\title{
Atopic Dermatitis in Older Adults: A Review of Treatment Options
}

\author{
Ryoji Tanei ${ }^{1}$ (D) \\ Published online: 21 February 2020 \\ (c) The Author(s) 2020
}

\begin{abstract}
Atopic dermatitis (AD) in older adults (elderly $\mathrm{AD}$ ) has recently emerged as a newly defined subgroup of $\mathrm{AD}$. When selecting treatment options, clinical characteristics of elderly $\mathrm{AD}$ and age-specific factors of older patients must be considered. As in other age groups, regular application of moisturizers in combination with topical corticosteroids and calcineurin inhibitors, adjunctive administration of oral antihistamines/anti-allergic drugs, and avoidance of exacerbating factors comprise basic treatments for elderly AD. For moderate-to-severe cases and/or in those with a decreased ability to use topical treatments, powerful anti-inflammatory treatments may become necessary as additional treatment options. While low-dose oral corticosteroids may be useful for cases of elderly AD, careful attention should be paid to adverse effects. Oral cyclosporine (ciclosporin) is less commonly used due to the increased risk of malignancy and organ toxicity in older patients with AD. Narrow-band ultraviolet B phototherapy may also be useful for older patients, although the necessity of frequent hospital visits for irradiation therapy may become a burden of disease for such patients. As a biologic, dupilumab therapy markedly improves skin lesions and itch in older patients with $\mathrm{AD}$, with a rapid response and non-serious adverse effects. Nevertheless, injection pain, expensive medical care, and regular follow-up every 2 weeks are disadvantages of dupilumab therapy. Therefore, clinicians must prioritize individualized treatment options that will reduce the burden of disease for cases of elderly AD.
\end{abstract}

\section{Key Points}

The prevalence of atopic dermatitis (AD) in older adults has increased and characteristics of elderly AD have become apparent.

Several factors must be considered when choosing therapies for older patients with AD.

Clinicians should prioritize treatment options that will reduce the burden of disease for older patients with $\mathrm{AD}$.
Ryoji Tanei

rtanei@aol.com

1 Department of Dermatology, Tokyo Metropolitan Geriatric Hospital and Institute of Gerontology, 35-2 Sakaecho, Itabashi, Tokyo 173-0015, Japan

\section{Introduction}

Atopic dermatitis (AD) is a chronic, relapsing pruritic skin disease involving allergic inflammation and skin barrier defects in relation to environmental stimuli and the patient's genetic background [1]. AD is generally considered a pediatric disease with a good prognosis; this condition is known as classic childhood $\mathrm{AD}$, as the onset of $\mathrm{AD}$ commonly begins in early infancy or childhood and resolution of AD typically occurs with increasing age until puberty. Although cases of AD either persisting or first appearing in adolescence and young adulthood have also been encountered (referred to as adolescent/adult $\mathrm{AD}$ ) [2], it was previously considered that the clinical manifestation of patients with adult $\mathrm{AD}$ would usually resolve with aging when patients reached their $50 \mathrm{~s}$ $[2,3]$. Thus, AD has been typically classified into infantile (age $<2$ years), childhood (age 2-12 years), and adolescent/ adult ( $>12$ years, but not over middle age) types according to patient age and characteristics of typical skin lesions [3]. However, the paradigm has recently changed. The number of cases of AD remaining or first appearing in adulthood [4, 5], and even in older adults [3,6], have increased and the characteristics of elderly AD have become apparent [7-10]. Hence, this newly defined subgroup of elderly AD (age $>60$ 
or $\geq 60$ years) was recently added into the classification of $\mathrm{AD}$ as a fourth subtype $[11,12]$. AD is now considered a lifelong condition in some populations.

With the increasing recognition of $\mathrm{AD}$ as a disease that affects older adults [12-18], it seems appropriate to review the drug treatment options for this condition in the elderly. In this article, treatment options, including topical, oral, and phototherapies as well as biologics, in the management of elderly AD are reviewed. Factors that need to be considered in choosing therapies for older patients with $\mathrm{AD}$ are also discussed.

\section{Clinical Characteristics of Atopic Dermatitis (AD) in Older Adults}

\subsection{Clinical Types and Age of Onset of Elderly AD}

In general, at least two types of AD have been identified: an immunoglobulin (Ig)E-allergic (extrinsic) type associated with high serum total IgE levels ( $>200$ or $400 \mathrm{IU} / \mathrm{L}$, according to the standards of the individual facility) and IgE-mediated sensitization to environmental allergens; and a non-IgE-allergic (intrinsic) type with normal serum total IgE levels and a lack of sensitization to environmental allergens $[11,19]$. Similar to other age groups, both the IgE-allergic and non-IgE-allergic types of $\mathrm{AD}$ occur in elderly $\mathrm{AD}$ when the diagnosis of $\mathrm{AD}$ is based on standardized diagnostic criteria [13]. In addition, as is the case with infantile $\mathrm{AD}$ [20], an indeterminate allergic type also occurs in elderly $\mathrm{AD}$, where the patient has normal serum total $\operatorname{IgE}$ levels and positivity for allergen-specific IgEs $[6,8,11]$; this type of $\mathrm{AD}$ is an intermediate, vague state between the IgE-allergic and non-IgE-allergic types. In contrast to infantile AD [1, 20], these three clinical phenotypes in elderly AD tend to be present in a discontinuous disease state and might reflect a different pathophysiology $[12,16]$.

The most common environmental allergens for IgEallergic elderly AD are house dust mites (HDMs; e.g., Dermatophagoides species), followed by pollens (e.g., grass pollen and Japanese cedar). Positivity rates in two studies were as follows: D. pteronyssinus $72 \%$, D. farinae $70.9 \%$, and grass pollen $68.4 \%$ in a Polish study [6]; and D. farinae $83.8 \%$ and Japanese cedar $56.8 \%$ in the study of our patients [8]. Positivity rates for food, fungus, and animal dander were relatively lower in these studies. Histopathological analyses revealed that as allergens for IgE-associated immediate, late-phase, and delayed-type hypersensitivities, HDMs may be involved in the pathophysiology of skin lesions of IgEallergic elderly $\mathrm{AD}[16,21]$.

The onset and clinical course of elderly $\mathrm{AD}$ are mainly as follows: senile onset (continuous form), senile recurrence with a history of classic childhood AD (outgrowth-recurrence form), and continuation and/or recurrence of adolescent/adult $\mathrm{AD}$ (both continuous and outgrowth-recurrence forms with a history of infantile-, childhood-, adolescent-, or adult-onset $\mathrm{AD})[1,8,12,16]$.

\subsection{Skin Manifestations}

Characteristic manifestations of $\mathrm{AD}$ differ according to the period of life. In the infantile phase, erythematous and papulovesicular eruptions, which frequently develop into oozing and crusting, are often observed on the face. In the childhood phase, atopic dry skin and lichenified flexural eczema of the extremities become more prominent. In the adolescent and young adult phases, chronic lichenified eczema on the upper part of the body predominates [3]. Skin manifestations in elderly $\mathrm{AD}$ basically match those of adolescent/young adult $\mathrm{AD}$ [22], but the reverse sign of lichenified eczema around unaffected folds of the elbows and knees is more common than the classic sign of localized lichenified eczema at those folds $[3,8,12,16]$. Localized lichenified eczema in the elbow and knee folds is a typical sign of classic childhood AD [23] and adolescent/young adult AD [2] but is uncommon in elderly AD, in which unaffected folds of the elbows and knees are more common $[8,10]$ and lichenified eczema surrounding the folds, particularly around the elbow folds, is frequently observed in the flexure and extensor sites of the extremities $[3,8]$. Other signs of AD such as refractory facial erythema (atopic red face), loss of the lateral eyebrows (Hertoghe sign), Dennie-Morgan infraorbital folds, and neck eczema with reticulate, ripple, or poikilodermic pigmentation (dirty neck) [22] are observed in moderate-tosevere cases. Nummular-form eczema, prurigo-form papules and nodules, and erythrodermic rash may also be observed $[12,16]$. In a cross-sectional, population-based study in the United States, it has been demonstrated that, compared with adult AD (18-59 years), clinical manifestations of elderly $\mathrm{AD}$ ( $\geq 60$ years) were associated with a significantly lower incidence of active lesions on the face and scalp, marginally significant lower incidence of flexural (including elbow and knee folds) lesions, and significantly higher incidence of lesions on the buttocks or genitals [10].

\subsection{Histopathology and Pathophysiology of Skin Lesions}

Histopathological evaluation of skin lesions in a patient with elderly AD is often needed for differential diagnosis from other chronic skin diseases such as cutaneous $\mathrm{T}$ cell lymphoma. Similar to adult AD, routine hematoxylin-eosin staining in elderly AD shows only non-specific chronic eczematous reactions with epidermal hyperplasia, mononuclear cell infiltration with eosinophils, and increased 
infiltration of mast cells in the upper dermis in lichenified eczema [3].

By immunohistochemical and molecular analyses, however, AD skin lesions are considered to be T cell-mediated biphasic immune reactions specific to environmental exposures such as allergens. Immune activation of $\mathrm{T}$ helper (Th) 2 (i.e., interleukin [IL]-4, IL-5, IL-13, and IL-31), Th22 (i.e., IL-22), and Th17 (i.e., IL-17) cytokines is involved in acute lesions, and progressive activation of Th2 and Th22 cytokines and significant upregulation of Th1 cytokines (i.e., interferon [IFN]- $\gamma$ ) are involved in chronic lesions [24-27]. Th 2 cytokines, i.e., IL- 4 and IL-13, stimulate B cells to produce IgE antibodies and promote IgE-induced upregulation of the high-affinity IgE receptor (FceRI) on mast cells and dendritic cells, and Th2 cytokine IL-5 induces peripheral eosinophil activation. Th2 and Th22 cytokines (i.e., IL-4, IL-13, and IL-22) contribute to the non-genetic impaired expression of skin barrier proteins (e.g., filaggrin) and barrier dysfunction of skin lesions. Th2 cytokine IL-31 is associated with causing itch, and Th22 cytokine IL-22 induces epidermal hyperplasia of chronic lesions in patients with $\mathrm{AD}$ [24-27].

In elderly $\mathrm{AD}$, phenotypic analyses of peripheral blood cytokine concentrations demonstrated the dominance of a Th2 cytokine profile, i.e., IL-4, IL-5, and IL-13, in older patients with IgE-allergic $\mathrm{AD}$, whereas dominance of a Th1 cytokine profile, i.e., IFN- $\gamma$, was observed in older patients with $\mathrm{AD}$ and low serum total $\mathrm{IgE}$ levels [6]. In a recent study on age-specific changes in the molecular phenotype of patients with $\mathrm{AD}$, serum total $\mathrm{IgE}$ levels and eosinophil counts were negatively correlated with age in patients with $\mathrm{AD}$, and patients with elderly $\mathrm{AD}$ tended to show immune skewing with decreased Th2 and Th22 cytokines and increased Th1 cytokines in lesional skin compared with patients with adult AD [9]. The participation and role of allergen-specific IgEs in the development of skin lesions of AD has been controversial [26], but immunohistopathological analysis in our previous studies indicated that, at least in patients with IgE-allergic elderly $\mathrm{AD}$, IgE-mediated allergic inflammation (composed of IgE-bearing cells, including mast cells, dermal dendritic cells, inflammatory dendritic epidermal cells, and Langerhans cells) accompanied by specific allergens (e.g., HDMs) play a critical role in the features of skin lesions [7, 16, 21].

\subsection{Diagnosis of Elderly AD}

For clinical diagnosis of elderly $\mathrm{AD}$, lichenified eczema in flexural sites of the extremities is the most important hallmark, although elbow and knee folds tend to be spared in elderly $\mathrm{AD}$. The existence of the pathognomonic clinical signs of AD discussed in Sect. 2.2 may also become a diagnostic hallmark. A personal and/or family history of atopic disorders (e.g., bronchial asthma, allergic rhinoconjunctivitis, and $\mathrm{AD}$ ) and elevated serum levels of total $\mathrm{IgE}$ and allergen-specific IgEs in response to environmental allergens would support the diagnosis. Long-term followup (i.e., $>6$ months) of the clinical course, analyses of the diagnostic features of AD using standardized criteria (e.g., the Hanifin and Rajka diagnostic criteria [28]), and exclusion of other pruritic skin conditions are thus required to diagnose elderly $\mathrm{AD}$ [13]. The main pruritic skin disorders necessitating differentiation from elderly $\mathrm{AD}$, especially from senile-onset or senile-recurrence types of elderly $\mathrm{AD}$, are as follows: asteatotic dermatitis, nummular dermatitis, contact dermatitis, chronic prurigo, adverse drug reactions, scabies, papuloerythroderma of Ofuji [29], and cutaneous T cell lymphoma [30]. However, certain disorders, including non-atopic eczema in the elderly [31], chronic eczematous eruption of aging [32], and senile erythroderma [33, 34], may be characterized by clinical findings similar to elderly $\mathrm{AD}$, and differential diagnosis from elderly $\mathrm{AD}$ may be difficult in some cases. Thus, it could be speculated that pruritic skin disorders in the elderly have etiological similarities to elderly $\mathrm{AD}[12,17]$ with regards to the impact of aging on pathogenic factors (e.g., physical skin barrier, innate and adaptive immunity, skin microbiota, and environmental stimuli) that characterize their symptoms and signs. In the near future, a greater understanding of the characteristics of elderly $\mathrm{AD}$ as a specific phenotype of $\mathrm{AD}$ in old age and clear criteria, including diagnostic biomarkers for definitive diagnosis, will be necessary for patients with elderly AD $[11,17]$.

\subsection{Complications of Coexisting/Underlying Disorders and Malignancy}

Most older patients with $\mathrm{AD}$ have some complications related to non-allergic coexisting/underlying disorders (e.g., hypertension) that markedly differ from the characteristics of younger age groups of AD. In our hospital, the incidence of coexisting/underlying disorders in cases of IgE-allergic AD was as follows: hypertension $58.3 \%$; heart disease $23.7 \%$; spinal disease $23.7 \%$; cerebrovascular disease $26.3 \%$; diabetes mellitus $18.4 \%$; and chronic renal disease $10.5 \%$ [8]. In recent cross-sectional studies in the United States, higher odds of non-allergic complications such as hypertension, heart diseases, diabetes, obesity, anxiety and depression, and autoimmune disease were observed in adult (including older adult) patients with $\mathrm{AD}$ than in control subjects [35], and osteoporosis was more frequently found in older patients with AD [36]. In addition, another recent study showed increased cardiovascular and atherosclerosis markers in blood of older patients with AD [18]. In clinical practice, the existence of these coexisting/underlying disorders may 
place restrictions on treatment options, such as the use of oral corticosteroids and cyclosporine (ciclosporin).

Meanwhile, the incidence of complicating malignancies in older patients with IgE-allergic AD might be lower than that in the general elderly population, although the relationship between AD and malignancy remains controversial [37]. In our retrospective analysis [8], the incidence of malignancy $(2.6 \%, n=1)$ among older patients with IgEallergic $\mathrm{AD}(n=38$; mean age $76.2 \pm 8.7$ years $)$ was significantly lower than that $(31.9 \%, n=23 ; p<0.05)$ in control subjects (older patients with benign epidermal cyst, $n=72$; mean age $74.7 \pm 7.0$ years). Furthermore, older patients with IgE-allergic AD tended to have a lower incidence of mortality from malignancy than control subjects. Although the limitations of retrospective analysis and the small sample size must be considered, these results suggest that the disease state in IgE-allergic elderly AD might involve immunological peculiarities acting against malignancy as a result of over-response against foreign substances, including selfantigens, in which cancer antigens could be involved [12, 16].

\section{Management of AD in Older Adults}

In the management of older patients with $\mathrm{AD}$, the aforementioned clinical characteristics of elderly AD (Sect. 2) and age-specific factors of patients described in this section must be considered when choosing treatment options, and the following should be evaluated: medical examination, e.g., severity and state of AD, general condition of the patient, past history and complications, and use of conventional drugs; laboratory tests, e.g., presence or absence of serum-specific IgEs and its classification strength against common environmental allergens [3, 8], serum levels of total $\mathrm{IgE}$ and thymus and activation-related chemokine (TARC; a biomarker of Th2 cytokine responses) [38], peripheral eosinophil counts, and routine peripheral blood testing for the screening of other latent internal disorders; and clinical examination, e.g., metal patch testing.

Treatment options for older patients with AD are summarized in Table 1.

\subsection{Basic Treatments}

In the treatment of elderly $\mathrm{AD}$, as in other age groups, management of elderly AD comprises treatment to maintain the skin barrier (e.g., moisturizers and emollients), antiinflammatory measures (e.g., topical corticosteroids, topical calcineurin inhibitors, and adjunctive oral antihistamines/ anti-allergic drugs), and the identification and avoidance of exacerbation factors (e.g., environmental allergens) following evaluation of the severity and disease state of patients with $\mathrm{AD}$ in accordance with the management guidelines [39-43] and systematic review of treatments [44] for AD. Hence, regular application of moisturizers in combination with topical corticosteroids and calcineurin inhibitors, adjunctive oral antihistamines/anti-allergic drugs, and avoidance of exacerbating factors are the basics of treatment [40] for elderly AD [12, 13, 16].

\subsubsection{Avoidance of Exacerbating Factors}

Environmental exposures to allergens (e.g., HDMs, pollens, and foods), irritants (e.g., inappropriate clothing, chemicals, and scratching) and pathogens (e.g., Staphylococcus aureus) have been implicated in the pathogenesis of $\mathrm{AD}$ [1, 24-27, 39-42]. Avoidance of these exacerbating factors (e.g., HDMs [45]) in the living space and lifestyle of patients with $\mathrm{AD}$ has been shown to improve AD symptoms [39-42, 44]. Sweat is one of the exacerbating factors for AD as a source of both allergens [46] and irritants [47]. Hence, washing away sweat by bathing and showering will lead to symptom improvement [39-42] and are also important for washing away other allergens, irritants, and pathogens on the skin surface. Therefore, therapeutic patient education would be useful in order to avoid exacerbating factors and modify problematic behaviors, such as habitual scratching [43, 44, 48]. In older patients with AD, however, avoidance of these environmental stimuli is not so easy because of their potentially decreased activities of daily living, cognitive function, and self-care ability [12]. For example, older individuals often have fewer baths and generally live in older homes, which have been suggested to have higher levels of HDM allergens, with a corresponding increased risk of exposure and sensitization to the allergens [49-51].

\subsubsection{Application of Moisturizers}

Dry skin (xerosis) is one of the main symptoms of AD and is also a feature of aging skin in older adults (age-related dry skin [senile xerosis]). Moreover, poor lifestyle habits such as excessive washing and showering sometimes worsen dry skin in older adults. Hence, gentle washing with a fully foaming soap is recommended, and rubbing with a towel should be avoided. Several experimental studies indicate that dry skin with an impaired skin barrier may become a cause of inflammation and itching in skin disorders, including AD [52]. Therefore, topical drugs with moisturizing effects should be used as a basic treatment to improve dry skin in patients with elderly AD. Topical moisturizers, e.g., urea or heparinoids, are first-choice drugs [40]. These moisturizers should be applied in combination with topical anti-inflammatory drugs (e.g., topical corticosteroids) immediately after bathing and showering, before the skin is completely dry. Moisturizers should be used on a wide range of skin 


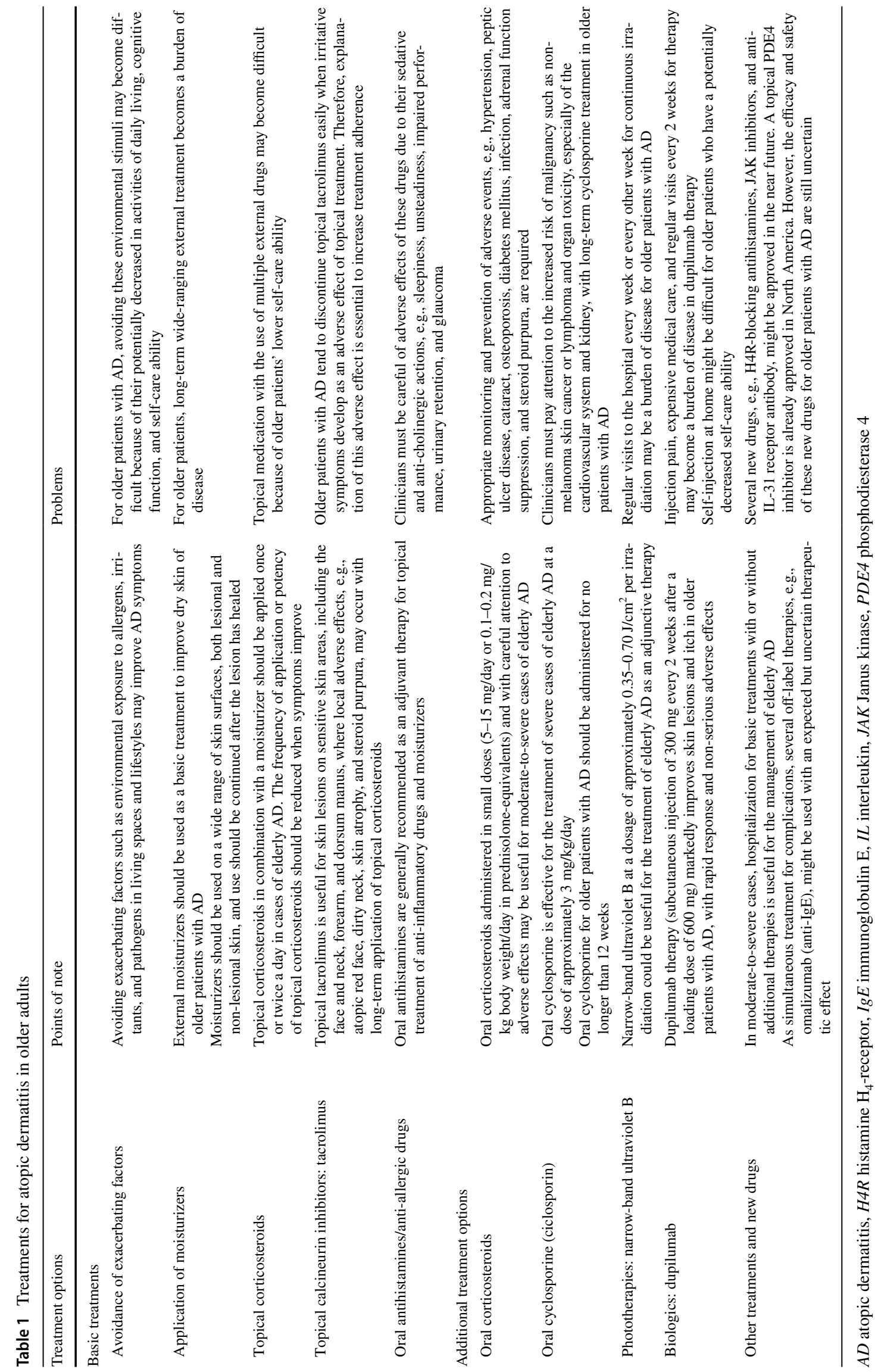


surfaces, both lesional and non-lesional skin, and use should be continued after the lesion has healed [15].

\subsubsection{Topical Corticosteroids}

Corticosteroids exert therapeutic effects by inhibiting transcription of several mediators (e.g., cytokines and adhesion molecules) from inflammatory cells in the skin lesion of $\mathrm{AD}$ as a result of binding to regulatory elements of many genes via their receptor [53].

Topical corticosteroids are used as a first-line antiinflammatory treatment for patients with $\mathrm{AD}$ and are effective for both active inflammation and disease prophylaxis. Several factors should be considered when choosing a corticosteroid, including potency, drug formulation, severity of skin lesions, and the body area to which the medication will be applied [39-42]. As a rule, low-potency (medium class or lower) topical corticosteroids should be used for skin lesions on the face and neck where the drug absorption rate is high. High-potency (very-strong class or lower) topical corticosteroids can be used for skin lesions on the body and extremities. However, use of super-potency (strongest) topical corticosteroids should be limited to only refractory lesions such as pruriginous nodules or severe lichenification. Clinicians must always be aware of skin problems (e.g., skin atrophy, telangiectasia, and steroid purpura) associated with the long-term use of topical corticosteroids, especially for older adults with already fragile skin [54]. Concerning the frequency of application, no significant difference was found in the improvement rate between once-daily and more frequent application of topical corticosteroids in several studies [44]. Therefore, the principal guidelines of AD management [39-42] recommended once- or twice-daily applications of topical corticosteroids in combination with a moisturizer to treat AD-related itch and skin lesions, with reduction in the application frequency or potency of topical corticosteroids when symptoms improve. It has been demonstrated that even low-potency topical corticosteroids can broadly improve immune and barrier responses in patients with moderate-to-severe AD [55]. In older patients, however, topical treatment with the use of multiple external drugs may become difficult because of patients' lower self-care ability. Therefore, to improve adherence to external treatment in older patients with $\mathrm{AD}$, it is sometimes necessary to prescribe topical corticosteroids and moisturizers as an admixed preparation, although the medicinal properties and stability of the drugs may be somewhat unstable [56].

\subsubsection{Topical Calcineurin Inhibitors}

Topical calcineurin inhibitors (tacrolimus and pimecrolimus; tacrolimus only in Japan) inhibit cytokine production by $\mathrm{T}$ cells via their intracellular calcineurin-inhibiting mechanism, which differs from that of topical corticosteroids. The therapeutic potency of $0.1 \%$ tacrolimus ointment is equivalent to topical corticosteroids with intermediate potency (strong class) [39-42]. Local adverse effects from long-term application of topical corticosteroids in sensitive or thin skin areas can cause atopic red face, dirty neck, skin atrophy, and steroid purpura [13]; however, these are rarely seen with long-term use of topical tacrolimus [39-42]. Therefore, topical tacrolimus is useful for skin lesions on the face and neck, forearm, and dorsum manus of older patients with AD. Topical tacrolimus is typically applied once daily up to a maximum frequency of twice a day. The most commonly observed adverse effect is irritative symptoms (e.g., transient burning sensation and hot flushes) at the application site during the initial few days after starting treatment [39-42]. Older patients with AD tend to readily discontinue tacrolimus application because of this adverse effect; therefore, prior explanation of this adverse effect is essential to improve treatment adherence. Irritative symptoms may be reduced by using a small amount of tacrolimus ointment mixed with an emollient (e.g., petrolatum), and the mixture may be useful for the initial period of the treatment. To date, it is considered that application of topical tacrolimus does not appear to increase the risk of skin cancer, lymphoma, or other malignancies with proper usage [39, 42].

\subsubsection{Oral Antihistamines/Anti-allergic Drugs}

Oral antihistamines (histamine $\mathrm{H}_{1}$-receptor antagonists) are widely used for $\mathrm{AD}$-related itch because several studies support a role of histamine in the pathogenesis of $\mathrm{AD}[57,58]$. In Japan, many study reports have discussed the positive effects of antihistamines on AD [39, 59, 60]. Conversely, in Europe and the United States, many reports have presented negative opinions [39, 41, 42, 61, 62]. Therefore, oral antihistamines are generally recommended as an adjuvant therapy to topical treatment with anti-inflammatory drugs and moisturizers [39] or as short-term sporadic use in patients with night-time itch [41, 42]. In older patients with $\mathrm{AD}$, however, oral antihistamines may become one of the basic treatments for AD-related itch [40] or are used as adjunctive therapy for topical treatment [59], because older patients usually require systemic anti-pruritic drugs for relief from persistent itch caused by insufficient topical treatment and/or the intractable disease state of AD. In Japanese guidelines for AD management, non-sedative second-generation antihistamines with low anti-cholinergic action are recommended as first-choice agents [39] because they have therapeutic anti-pruritic effects that are similar to those of sedative antihistamines [39,60]. Additional administration of sedative first-generation antihistamines, which have inhibitory effects on the central nervous system, before bedtime is sometimes useful for older patients with $\mathrm{AD}$ who 
experience sleep disturbance caused by severe night-time itch [40-42, 63]. When using oral antihistamines, especially first-generation antihistamines, clinicians must be aware of adverse effects due to their sedative and anti-cholinergic actions, e.g., sleepiness, unsteadiness, impaired performance, urinary retention, and glaucoma.

Combined use of oral antihistamines and anti-allergic drugs without an antihistamine effect may also be effective for itch and related skin lesions in older patients with AD. Disodium cromoglycate, an anti-allergic agent used for food sensitization and/or systemic metal allergy [64, 65], is effective in patients with positivity for specific IgEs to food allergens or metal patch testing $[6,8,19]$. Suplatast tosilate (a Th2 cytokine inhibitor) has a therapeutic effect by inhibiting Th2 cytokine production and decreasing eosinophils at inflammatory sites of skin lesions in patients with raised serum levels of total and specific IgEs and/or TARC [66].

\subsection{Additional Treatment Options}

The efficacy of basic treatments tends to be inadequate in older patients with $\mathrm{AD}$ because avoiding exacerbating factors in the environment and applying sufficient topical medication may become difficult with common geriatric problems and lifestyle changes, e.g., potentially decreased self-care ability with aging and isolation. For older patients, longterm, wide-ranging external treatment becomes a burden of disease. In addition, it has become apparent that moderateto-severe cases of AD have additional features of systemic inflammation [18] and autoallergy in which T cell-mediated specific immune pathways react not only to environmental allergens but also to self-proteins in the skin as autoantigens $[1,25,67]$. Therefore, for moderate-to-severe cases and/or patients with a lower ability to use topical treatments, powerful anti-inflammatory treatments (e.g., oral corticosteroids, cyclosporine, phototherapy, and biologics) may become necessary as additional treatment options [53, 68].

\subsubsection{Oral Corticosteroids}

Unlike in childhood/adolescent AD, in which adverse effects such as growth suppression are of concern, oral corticosteroids may be relatively easy to use in cases of elderly AD as an additional therapy to topical corticosteroids. Oral corticosteroids are generally recommended as short-term relief for patients with moderate-to-severe AD by systematic review [69], expert opinion [68, 70], and the guidelines for AD [39-41, 43]. However, for the reasons mentioned in Sect. 3.2, they tend to be prescribed at low doses for mid- to long-term use for the treatment of elderly AD. In our retrospective analyses, the usefulness of oral corticosteroids $(5-15 \mathrm{mg} /$ day or $0.1-0.2 \mathrm{mg} / \mathrm{kg}$ body weight/day in prednisolone-equivalents) in combination with basic treatments was evident in the treatment for elderly AD $[8,12,16]$. In these analyses, oral corticosteroid therapy combined with basic treatments was applied for 27 cases of moderate-to-severe elderly $\mathrm{AD}$ (IgE-allergic $\mathrm{AD}, n=16$; indeterminate-allergic $\mathrm{AD}, n=6$; non-IgE-allergic $\mathrm{AD}$, $n=5$ ), and the mean \pm standard deviation Objective Severity Scoring of Atopic Dermatitis for each clinical type was $41.6 \pm 19.1,22.5 \pm 8.0$, and $37.7 \pm 21.7$, respectively. Using a mean ( \pm standard deviation) dosage of oral corticosteroids of $7.7 \pm 4.1 \mathrm{mg} / \mathrm{day}(8.6 \pm 4.1,5.0 \pm 3.1$, and $7.5 \pm 1.8 \mathrm{mg} /$ day in prednisolone-equivalents for each clinical type, respectively), the prognosis of these cases for a duration of 6 months or more was observed to be clinical improvement, defined as disappearance of skin lesions in $>95 \%$ of lesional areas with combined therapy at a dosage of oral corticosteroids of $\leq 5 \mathrm{mg} /$ day prednisolone-equivalents, in $68.8 \%$ of IgE-allergic $\mathrm{AD}, 100 \%$ of indeterminate-allergic $\mathrm{AD}$, and $80 \%$ of non-IgE-allergic AD cases. Furthermore, clinical remission, defined as the same disappearance of skin lesions even after discontinuation of oral corticosteroids, was also observed in $18.8 \%$ of IgE-allergic $\mathrm{AD}, 50 \%$ of indeterminate-allergic $\mathrm{AD}$, and $40 \%$ of non-IgE-allergic $\mathrm{AD}$ cases [8]. Although the evidence level of our observational study was very low, oral corticosteroid therapy with basic treatments may become an effective treatment option for elderly AD.

However, there are some important notes for the usage of oral corticosteroid therapy. It should be used in combination with appropriate basic treatments and sufficient evaluation of exacerbating factors and the state of patients with AD. The dosage should be decreased as symptoms improve, and appropriate monitoring and prevention of adverse events such as hypertension, peptic ulcer disease, cataract, osteoporosis, diabetes, infections, adrenal function suppression, and steroid purpura are needed. Working knowledge of the adverse effects of oral corticosteroids is necessary and precautions are required. For example, oral betamethasone $0.5 \mathrm{mg} /$ day (almost equal potency to prednisolone $4.2 \mathrm{mg} /$ day [71]) has a risk of adrenal suppression equivalent to external use of a super-potency (strongest) topical corticosteroid, i.e., $0.05 \%$ clobetasol 17-propionate ointment in quantity of $10 \mathrm{~g} /$ day $[72,73]$. In addition, mid- to long-term use ( $\geq 3$ months) of oral corticosteroids as $>2.5 \mathrm{mg} /$ day prednisolone-equivalents may increase the risk of fracture [74, 75]; hence, preventive administration of bisphosphonates or an active vitamin $\mathrm{D}_{3}$ analog for corticosteroid-induced osteoporosis is needed in these patients [75]. To prevent hepatitis B (HB) virus reactivation during immunosuppressive drug therapy including oral corticosteroids, blood screening for HB surface antigen (HBsAg) and antibody to $\mathrm{HB}$ core antigen (anti-HBcAb) should be carried out, and patients with positive results for HBsAg and/ or anti-HBcAb must be treated with preventive measures based on the guidelines for hepatic disorders [76]. Vascular 
strengthening drugs such as vitamin $\mathrm{C}$ preparation [77] and [carbazochrome sodium [78] may be useful for the treatment of steroid purpura, which may be more likely to occur in older patients with $\mathrm{AD}$ as it is a similar condition to senile purpura [79].

\subsubsection{Oral Cyclosporine}

Cyclosporine inhibits $\mathrm{T}$ cell-dependent cytokine products and immune pathways by blocking nuclear factor of activated $T$ cells [53]. Oral cyclosporine is also effective for the treatment of severe cases of elderly $\mathrm{AD}$ when administered at an optimal and sufficient dose (e.g., $3 \mathrm{mg} / \mathrm{kg} /$ day) [39, 80]. However, clinicians must pay attention to long-term use of cyclosporine in older patients with $\mathrm{AD}$ due to the potential increased risks of malignancy such as non-melanoma skin cancer or lymphoma $[81,82]$ and organ toxicity, especially in the cardiovascular system and kidney $[43,80$, 83]. Therefore, the duration of use of oral cyclosporine for older patients with $\mathrm{AD}$ should be no longer than 12 weeks, although it could be restarted intermittently after withdrawal of 2 weeks or more [39]. In general, the risk of carcinogenesis increases with aging [84], and renal dysfunction is common in older individuals. Thus, older people are more likely to be concerned about these adverse effects of oral cyclosporine. Unfortunately, after stopping the use of oral cyclosporine, relapse of symptoms is frequently observed in elderly AD. Furthermore, the price of cyclosporine is much higher than that of oral corticosteroids. Therefore, oral cyclosporine may be less commonly used in the treatment of elderly AD.

\subsubsection{Phototherapy}

As described in Sect. 3.2, powerful anti-inflammatory treatments such as oral corticosteroids or cyclosporine in combination with basic treatments may be needed for the management of elderly $\mathrm{AD}$; however, underlying conditions (e.g., diabetes, hypertension, heart diseases, renal dysfunction, and medical history of hepatitis or tuberculosis) in older patients may preclude the use of such treatments. In such cases, narrow-band ultraviolet B (UVB; wavelength $311 \pm 2 \mathrm{~nm}$ ) at a dosage of approximately $0.35-0.70 \mathrm{~J} / \mathrm{cm}^{2}$ per irradiation could be useful for the treatment of elderly $\mathrm{AD}$ (Fig. 1) as an adjunctive therapy [85]. The immunomodulatory effects of narrow-band UVB phototherapy for AD are achieved via suppression of immune pathways of Th2, Th22, and Th1 cells and their cytokines in the lesional skin of $\mathrm{AD}$ [86]. Of note, narrow-band UVB phototherapy may not be effective for treating acute exacerbations of $\mathrm{AD}$, and overdose of irradiation may induce eczema flare-ups $[68,85]$. Slight irritation and mild diffuse pigmentation or solar lentigo may occur after irradiation as adverse effects of narrow-band UVB [85]. In principle, narrow-band UVB phototherapy should not be combined with oral cyclosporine or topical calcineurin inhibitors because the risk of skin cancer may increase with this combination treatment $[53,68]$. Hospitalization may be required for phototherapy irradiation of more than three times a week as an initial treatment in older patients with AD. Patients must visit the hospital every week or every other week for continuous irradiation as an outpatient in a therapeutic period from weeks to months, and this may represent a burden of disease for older patients with $\mathrm{AD}[42,85]$.

\subsubsection{Biologics}

At present, dupilumab is the only approved biologic for the treatment of AD. Dupilumab is a fully human monoclonal antibody against the $\alpha$-subunit of IL-4 receptors that blocks signaling from both IL-4 and IL-13, which are key Th2 cytokines in the pathophysiology of $\mathrm{AD}[25,27,43,53,68]$. Subcutaneous injection therapy of dupilumab ( $300 \mathrm{mg}$ every 2 weeks after a loading dose of $600 \mathrm{mg}$ ) markedly improves skin lesions and itch in adult patients with moderate-tosevere $\mathrm{AD}$ with a rapid response and non-serious adverse effects [87-90]. Good efficacy for adult and older patients with AD (Fig. 1) has been reported, along with decreased expression of genes involved in Th2-associated cytokines/ chemokines, epidermal hyperplasia, T cells, dendritic cells, and Th17/Th22 activity in the lesional skin after therapy [89, 90]. Dupilumab could be used as a substitute therapy for oral corticosteroids or cyclosporine in patients with inadequate response or adverse effects following application of those therapies [91]. Dupilumab is also useful for patients with complications of other Th2-immunopathway disorders such as bronchial asthma [92]. The effectiveness of dupilumab in the treatment of other pruritic skin disorders in older adults such as idiopathic chronic eczematous eruption of aging [32] may indicate the existence of common Th2-immunodominant etiological backgrounds in elderly $\mathrm{AD}$ and other pruritic skin disorders.

Major disadvantages of dupilumab treatment are conjunctivitis $[93,94]$ and inadequate efficacy for residual erythematous dermatitis on the face and neck [95-97]; however, the former can be successfully treated with fluorometholone $0.1 \%$ eye drops or tacrolimus $0.03 \%$ eye ointment $[92,93]$ and the latter may improve with combined antifungal therapy (e.g., topical ketoconazole or oral itraconazole) or topical tacrolimus with allergen avoidance (e.g., shampoo) under expecting a therapeutic effect on paradoxical worsening of hepersensitivity (probably Th1 hyper-response caused by the suppression of Th2 immunity) to Malassezia species or environmental contact allergens [95-97]. The burden of disease in dupilumab therapy for older patients with AD may be injection pain, expensive medical care, and regular visits 

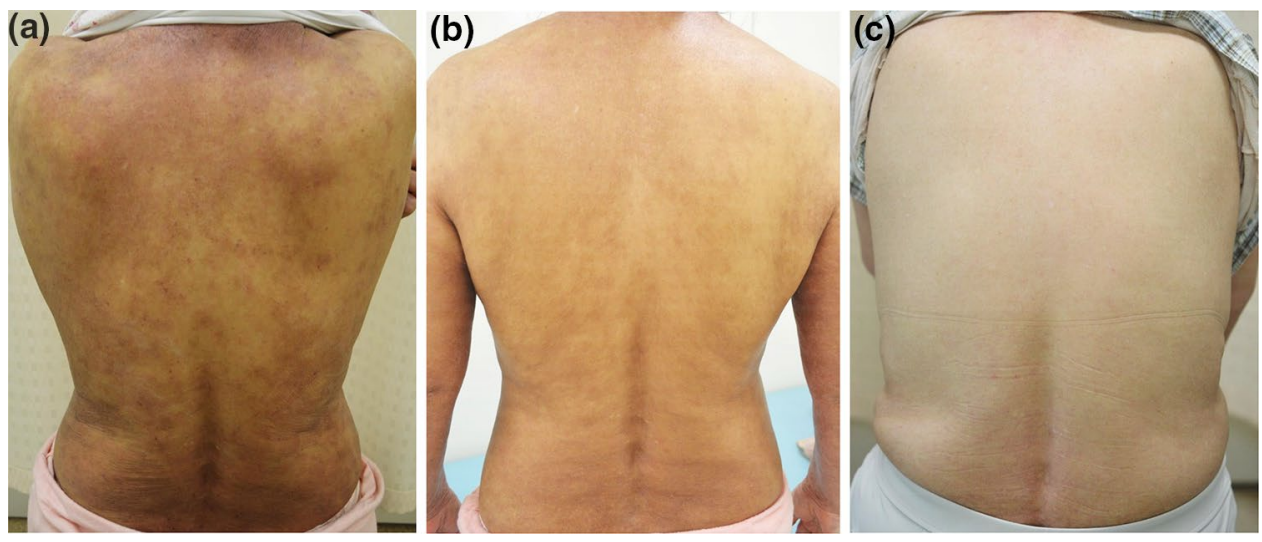

Fig. 1 Clinical course in an older female patient with refractory atopic dermatitis after the combined use of basic treatments and additional therapies. a Skin manifestation at the exacerbation period (immediately before the start of phototherapy) at 61 years of age (EASI 29.4; laboratory data: total IgE $9410 \mathrm{IU} / \mathrm{mL}$, TARC $5736 \mathrm{pg} /$ $\mathrm{mL}$, and peripheral eosinophil count $2070 \mu \mathrm{L})$. b After narrow-band ultraviolet B phototherapy (4 weeks after starting phototherapy)

every 2 weeks for the therapy. Home self-injections of the expensive and somewhat painful biologics might be difficult for older patients with $\mathrm{AD}$ who have potentially decreased self-care ability.

\subsection{Other Treatments and New Drugs}

In moderate-to-severe cases, hospitalization for basic treatments with or without additional therapies is useful in the management of elderly AD.

Oral administration of antimicrobial drugs is not effective for $\mathrm{AD}$ treatment without signs of infection; therefore, oral antibiotics are recommended for use only in cases with obvious clinical signs of infection [39, 43]. Antiviral therapy is also needed for patients with $\mathrm{AD}$ and herpes simplex infection at the time of viral activation with vesicular formation on the lips and pubic area $[43,53]$. Interestingly, patients with elderly $\mathrm{AD}$ and herpes simplex infection might have a decreased tendency to develop a widespread severe form of the infection, i.e., eczema herpeticum; however, the cause of this is unknown.

There are several off-label therapies that might be used for older patients with $\mathrm{AD}$ as simultaneous treatment for complications (e.g., urticaria, bronchial asthma, allergic rhinitis, or rheumatic disorders), with an expected but uncertain therapeutic effect, e.g., omalizumab (anti-IgE), rituximab (anti-cluster-of-differentiation [CD] 20), and ustekinumab (anti-IL-12/IL-23) as biologics, methotrexate and azathioprine as immunosuppressants, and a sublingual agent of the HDM extract as allergen-specific immunotherapy [27, 43, $53,68]$. at 62 years of age (EASI 17.6; laboratory data: not tested). c After dupilumab therapy (1 year after starting subcutaneous injection therapy) at 66 years of age (EASI 3.5; laboratory data: total IgE $2890 \mathrm{IU} /$ $\mathrm{mL}$, TARC $377 \mathrm{pg} / \mathrm{mL}$, and peripheral eosinophil count $421 \mu \mathrm{L}$ ). EASI Eczema Area and Severity Index, $I g E$ immunoglobulin E, TARC thymus and activation-related chemokine

In addition, global approval of several new drugs is expected in the near future, including anti-pruritic drugs such as histamine $\mathrm{H}_{4}$ receptor-blocking antihistamines, small molecules such as phosphodiesterase (PDE) 4 inhibitors and Janus kinase inhibitors, and biologics such as anti-IL-31 receptor antibody. In the United States and Canada, topical crisaborole (a novel PDE4 inhibitor) is already approved and available as a new option for patients with mild-to-moderate $\mathrm{AD}$ [98]. These treatments are currently being evaluated for efficacy and safety in worldwide clinical trials in patients with moderate-to-severe $\operatorname{AD}[25-27,43,99,100]$. With the advent of these new drugs, the management of $\mathrm{AD}$ is anticipated to improve.

\section{Conclusions}

Although AD is not a life-threatening disease, it brings severe disruption to the quality of life of older patients with $\mathrm{AD}$, who are in their last stage of life. In a retrospective analysis, it was found that the majority of older patients who died had continued to receive treatments for AD until just before death [8]. With current treatment options, complete remission (outgrowth, a condition without any need for treatment) is rarely achieved in elderly $\mathrm{AD}$ and, thus, most older patients reach the end of their life with AD.

In the near future, personalized care for patients with $\mathrm{AD}$ is anticipated, with targeted therapeutics utilizing differential strategies based on clinical phenotypes and endotypes of $\mathrm{AD}$, biomarkers and molecular analyses, and co-morbidities and complications of patients $[1,11,18,24,26,27,100]$. 
Clinicians must prioritize treatments that reduce the burden of disease in older patients with AD.

\section{Compliance with Ethical Standards}

Funding This research received no specific grant from any funding agency in the public, commercial, or not-for-profit sector.

Conflict of interest The authors declare no conflict of interest.

Ethics approval The Medical Ethics Committee of Tokyo Metropolitan Geriatric Hospital and Institute of Gerontology gave approval to conduct this study (No.260301).

Open Access This article is licensed under a Creative Commons Attribution-NonCommercial 4.0 International License, which permits any non-commercial use, sharing, adaptation, distribution and reproduction in any medium or format, as long as you give appropriate credit to the original author(s) and the source, provide a link to the Creative Commons licence, and indicate if changes were made. The images or other third party material in this article are included in the article's Creative Commons licence, unless indicated otherwise in a credit line to the material. If material is not included in the article's Creative Commons licence and your intended use is not permitted by statutory regulation or exceeds the permitted use, you will need to obtain permission directly from the copyright holder.To view a copy of this licence, visit http://creativecommons.org/licenses/by-nc/4.0/.

\section{References}

1. Bieber T. Personalized management of atopic dermatitis: beyond emollients and topical steroids. In: Bieber T, Nestle F, editors. Personalized treatment options in dermatology. Berlin: Springer; 2015. p. 61-76.

2. Sulzberger MB, Goodman J. The relative importance of specific skin hypersensitivity in adult atopic dermatitis. JAMA. 1936;106:1000-3.

3. Tanei R, Katsuoka K. Clinical analyses of atopic dermatitis in the aged. J Dermatol. 2008;35:562-9.

4. Margolis JS, Abuabara K, Bilker W, Hoffstad O, Margolis DJ. Persistence of mild to moderate atopic dermatitis. JAMA Dermatol. 2014;150(6):593-600.

5. Bannister MJ, Freeman S. Adult-onset atopic dermatitis. Aust J Dermatol. 2000;41:225-8.

6. Bozek A, Fisher A, Filipowska B, Mazur B, Jazab J. Clinical features and immunological markers of atopic dermatitis in elderly patients. Int Arch Allergy Immunol. 2012;157:372-8.

7. Tanei R, Hasegawa Y, Sawabe M. Abundant immunoglobulin E-positive cells in skin lesions support an allergic etiology of atopic dermatitis in the elderly. J Eur Acad Dermatol Venereol. 2013;27:952-60.

8. Tanei R. Clinical characteristics, treatments, and prognosis of atopic eczema in the elderly. J Clin Med. 2015;4:979-97.

9. Zhou L, Leonard A, Pavel AB, Malik K, Raja A, Glickman J, et al. Age-specific changes in the molecular phenotype of patients with moderate-to-severe atopic dermatitis. J Allergy Clin Immunol. 2019;144(1):144-56.

10. Silverberg JI, Margolis DJ, Boguniewicz M, Fonacier L, Grayson MH, Ong PY, et al. Distribution of atopic dermatitis lesions in United States adults. J Eur Acad Dermatol Venereol. 2019;33(7):1341-8

11. Bieber T, D'Erme AM, Akdis CA, Traidl-Hoffmann C, Lauener $\mathrm{R}$, Schäppi G, et al. Clinical phenotypes and endophenotypes of atopic dermatitis: where are we, and where should we go? J Allergy Clin Immunol. 2017;139:S58-64.

12. Tanei R, Hasegawa Y. Atopic dermatitis in older adults: a viewpoint from geriatric dermatology. Geriatr Gerontol Int. 2016;16(Suppl. 1):75-86.

13. Tanei R. Atopic dermatitis in the elderly. Inflamm Allergy Drug Targets. 2009;8:394-404.

14. Katsarou A, Armenaka MC. Atopic dermatitis in older patients: particular points. J Eur Acad Dermatol Venereol. 2011;25:12-8.

15. Irawan Y, Rihatmadja R, Legiawait L, Yusharyahya SN, Sularsito SA. Atopic dermatitis in the elderly. J Gen Pro DVI. 2016;1:54-61.

16. Tanei R. Senile atopic dermatitis. In: Katayama I, Murota H, Satoh T, editors. Evolution of atopic dermatitis in the 21st century. Singapore: Springer; 2018. p. 229-48.

17. Williamson S, Merritt J, De Benedetto A. Atopic dermatitis in the elderly: a review of clinical and pathophysiological hallmarks. Br J Dermatol. 2020;182(1):47-54.

18. He H, Li R, Choi S, Zhou L, Pavel A, et al. Increased cardiovascular and atherosclerosis markers in blood of older patients with atopic dermatitis. Ann Allergy Asthma Immunol. 2020;124:70-8.

19. Tokura Y. Extrinsic and intrinsic types of atopic dermatitis. J Dermatol Sci. 2010;58:1-7.

20. Park JH, Choi YL, Namkung JH, Kim WS, Lee JH, Park HJ, et al. Characteristics of extrinsic and intrinsic atopic dermatitis in infancy: correlations with laboratory variables. $\mathrm{Br}$ J Dermatol. 2006;155:778-83.

21. Tanei R, Hasegawa Y. Double-positive immunoglobulin $\mathrm{E}+$ and Dermatophagoides farinae antigen + dendritic cells are observed in skin lesions of older adults with atopic dermatitis: an immunohistological study. Dermatol Clin Res. 2017;3(1):134-50.

22. Nishioka K. Atopic eczema of adult type in Japan. Aust J Dermatol. 1996;37(Suppl):S7-9.

23. Aoki T, Fukuzumi T, Adachi J, Endo K, Kojima M. Re-evaluation of skin lesion distribution in atopic dermatitis. Analysis of cases 0 to 9 years of age. Acta Derm Venereol Suppl (Stockh). 1992;176:19-23.

24. Leung DY, Guttman-Yassky E. Deciphering the complexities of atopic dermatitis: shifting paradigms in treatment approaches. J Allergy Clin Immunol. 2014;134:769-79.

25. Furue M, Chiba T, Tsuji G, Ulzii D, Kido-Nakahara M, Nakahara $\mathrm{T}$, et al. Atopic dermatitis: immune deviation, barrier dysfunction, $\operatorname{IgE}$ autoreactivity and new therapies. Allergol Int. 2017;66:398-403.

26. Werfel T, Allam JP, Biedermann T, Eyerich K, Gilles S, Guttman-Yassky E, et al. Cellular and molecular immunologic mechanisms in patients with atopic dermatitis. Allergy Clin Immunol. 2016;138:336-49.

27. Paller AS, Kabashima K, Bieber T. Therapeutic pipeline for atopic dermatitis: end of the drought? J Allergy Clin Immunol. 2017;140:633-43.

28. Hanifin JM, Rajka G. Diagnostic features of atopic dermatitis Acta Derm Venerol (Stockh). 1980;92:44-7.

29. Camacho FM, García-Hernandez MJ, Muñoz-Pérez MA, Mazuecos J, Sotillo I. Ofuji papuloerythroderma in elderly woman with atopic erythroderma. J Eur Acad Dermatol Venereol. 2001;15:264-6.

30. Miyagaki T, Sugaya M. Erythrodermic cutaneous T-cell lymphoma: how to differentiate this rare disease from atopic dermatitis. J Dermatol Sci. 2011;64:1-6. 
31. Hüls A, Abramson M, Sugiri D, Fuks K, Krämer U, Krutmann $\mathrm{J}$, et al. Nonatopic eczema in elderly women: effect of air pollution and genes. J Allergy Clin Immunol. 2019;143:378-85.

32. Brummer GC, Wang LT, Sontheimer RD. A possible role for dupilumab (Dupixent) in the management of idiopathic chronic eczematous eruption of aging. Dermatol Online J. 2018;24(2):3.

33. Asai T, Horiuchi Y. Senile erythroderma with serum hyper IgE. Int J Dermatol. 1989;28:255-8.

34. Nakano-Tahara M, Terao M, Nishioka M, Kitaba S, Murota H, Katayama I. T helper 2 polarization in senile erythroderma with elevated levels of TARC and IgE. Dermatology. 2015;230:62-9.

35. Silverberg JI, Gelfand JM, Margolis DJ, Boguniewicz M, Fonacier L, Grayson MH, et al. Association of atopic dermatitis with allergic, autoimmune, and cardiovascular comorbidities in US adults. Ann Allergy Asthma Immunol. 2018;121:604-12.

36. Shaheen MS, Silverberg JI. Atopic dermatitis is associated with osteoporosis and osteopenia in older adults. J Am Acad Dermatol. 2019;80:550-1.

37. Brunner PM, Silverberg JI, Guttman-Yassky E, Paller AS, Kabashima K, Amagai M, et al. Increasing comorbidities suggest that atopic dermatitis is a systemic disorder. J Investig Dermatol. 2017;137:18-25.

38. Kataoka Y. Thymus and activation-regulated chemokine as a clinical biomarker in atopic dermatitis. J Dermatol. 2014;41:221-9.

39. Katoh N, Ohya Y, Ikeda M, Ebihara T, Katayama I, Saeki H, et al. Clinical practice guidelines for the management of atopic dermatitis 2018. J Dermatol. 2019;46:1053-101.

40. Katayama I, Aihara M, Ohya Y, Saeki H, Shimojo N, et al. Japanese guidelines for atopic dermatitis 2017. Allergo Int. 2017;66:230-47.

41. Eichenfield LF, Ahluwalia J, Waldman A, Borok J, Udkoff J, Boguniewicz M. Current guidelines for the evaluation and management of atopic dermatitis: a comparison of the Joint Task Force Practice Parameter and American Academy of Dermatology guidelines. J Allergy Clin Immunol. 2017;139(4S):S49-57.

42. Wollenberg A, Barbarot S, Bieber T, Christen-Zaech S, Deleuran M, Fink-Wagner A, European Dermatology Forum (EDF), the European Academy of Dermatology and Venereology (EADV), the European Academy of Allergy and Clinical Immunology (EAACI), the European Task Force on Atopic Dermatitis (ETFAD), European Federation of Allergy and Airways Diseases Patients' Associations (EFA), the European Society for Dermatology and Psychiatry (ESDaP), the European Society of Pediatric Dermatology (ESPD), Global Allergy and Asthma European Network (GA2LEN) and the European Union of Medical Specialists (UEMS), et al. Consensus-based European guidelines for treatment of atopic eczema (atopic dermatitis) in adults and children: part I. J Eur Acad Dermatol Venereol. 2018;32:657-82.

43. Wollenberg A, Barbarot S, Bieber T, Christen-Zaech S, Deleuran M, Fink-Wagner A, European Dermatology Forum (EDF), the European Academy of Dermatology and Venereology (EADV), the European Academy of Allergy and Clinical Immunology (EAACI), the European Task Force on Atopic Dermatitis (ETFAD), European Federation of Allergy and Airways Diseases Patients' Associations (EFA), the European Society for Dermatology and Psychiatry (ESDaP), the European Society of Pediatric Dermatology (ESPD), Global Allergy and Asthma European Network (GA2LEN) and the European Union of Medical Specialists (UEMS), et al. Consensus-based European guidelines for treatment of atopic eczema (atopic dermatitis) in adults and children: part II. J Eur Acad Dermatol Venereol. 2018;32:850-78.

44. Hoare C, Po ALW, Williams H. Systematic review of treatments for atopic eczema. Health Technol Assess. 2000;4(37):1-191.
45. Tan BB, Weald D, Strickland I, Freidmann PS. Double-blind controlled trial of effect of housedust-mite allergen avoidance on atopic dermatitis. Lancet. 1996;347(8993):15-8.

46. Hide M, Tanaka T, Yamamura Y, Koro O, Yamamoto S. IgE-mediated hypersensitivity against human sweat antigen in patients with atopic dermatitis. Acta Derm Venereol. 2002;82:335-40.

47. Murota H, Katayama I. Exacerbating factors of itch in atopic dermatitis. Allergol Int. 2017;66:8-13.

48. Barbarot S, Stalder JF. Therapeutic patient education in atopic eczema. Br J Dermatol. 2014;170:44-8.

49. Teplitsky V, Mumcuoglu KY, Babai I, Dalal I, Cohen R, Tanay A. House dust mites on skin, clothes, and bedding of atopic dermatitis patients. Int J Dermatol. 2008;47(8):790-5.

50. Yasueda H, Saito A, Nishioka K, Kutsuwada K, Akiyama K. Measurement of Dermatophagoides mite allergens on bedding and human skin surfaces. Clin Exp Allergy. 2003;33:1654-8.

51. Simpson A, Simpson B, Custovic A, Cain G, Craven M, Woodcock A. Household characteristics and mite allergen levels in Manchester, UK. Clin Exp Allergy. 2002;32:1413-9.

52. Yosipovitch G. Dry skin and impairment of barrier function associated with itch—new insights. Int J Cosmet Sci. 2004;26(1):1-7.

53. Simon D, Bieber T. Systemic therapy for atopic dermatitis. Allergy. 2014;69:46-55.

54. Katoh N, Tennstedt D, van Kan AG, Saint Aroman M, Loir A, Bacqueville D, et al. Gerontodermatology: the fragility of the epidermis in older adults. J Eur Acad Dermatol Venereol. 2018;32(Suppl 4):1-20.

55. Brunner PM, Khattri S, Garcet S, Finney R, Oliva M, Dutt R, et al. A mild topical steroid leads to progressive anti-inflammatory effects in the skin of patients with moderate-to-severe atopic dermatitis. J Allergy Clin Immunol. 2016;138:169-78.

56. Suzuki T, Uchino T, Miyazaki Y, Kagawa Y. The effect of storage time on the release profile of dexamethasone dipropionate from admixtures of steroid and heparinoid ointment. Pharmazie. 2014;69:104-8.

57. Butler JM, Chan SC, Stevens S, Hanifin JM. Increased leukocyte histamine release with elevated cyclic AMP-phosphodiesterase activity in atopic dermatitis. J Allergy Clin Immunol. 1983;71:490-7.

58. Ikoma A, Rukwied R, Ständer S, Steinhoff M, Miyachi Y, Schmelz M. Neuronal sensitization for histamine-induced itch in lesional skin of patients with atopic dermatitis. Arch Dermatol. 2003;139:1455-8.

59. Kawashima M, Tango T, Noguchi T, Inagi M, Nakagawa $\mathrm{H}$, Harada S. Addition of fexofenadine to a topical corticosteroid reduces the pruritus associated with atopic dermatitis in a 1-week randomized, multicentre, double-blind, placebo-controlled, parallel-group study. Br J Dermatol. 2003;148:1212-21.

60. Kawashima M, Furue M, Hide M, et al. A comparative study of anti-pruritic effects and the occurrence of drowsiness between sedating and non-sedating antihistamines (ACROSS trial) (in Japanese). J Clin Ther Med. 2011;27:563-73.

61. van Zuuren EJ, Apfelbacher CJ, Fedorowicz Z, Jupiter A, Matterne U, Weisshaar E. No high level evidence to support the use of oral $\mathrm{H} 1$ antihistamines as monotherapy for eczema: a summary of a Cochrane systematic review. Syst Rev. 2014;3:25.

62. Matterne U, Böhmer MM, Weisshaar E, Jupiter A, Carter B, Apfelbacher CJ. Oral H1 antihistamines as 'add-on' therapy to topical treatment for eczema. Cochrane Database Syst Rev. 2019;1:CD012167.

63. Haas HL, Sergeeva OA, Selbach O. Histamine in the nervous system. Physiol Rev. 2008;88:1183-241.

64. Paganelli R, Levinsky RJ, Brostoff J, Wraith DG. Immune complexes containing food proteins in normal and atopic subjects 
after oral challenge and effect of sodium cromoglycate on antigen absorption. Lancet. 1979;1(8129):1270-2.

65. Pigatto PD, Gibelli E, Fumagalli M, Bigardi A, Morelli M, Altomare GF. Disodium cromoglycate versus diet in the treatment and prevention of nickel-positive pompholyx. Contact Dermat. 1990;22:27-31.

66. Gorai S, Terada N, Kobayashi T, Nomura T, Kim WJ, Onai N, et al. The effect of suplatast tosilate on TARC production in peripheral blood mononuclear cells and TARC plasma levels. Allergo Int. 2005;54:373-80.

67. Hradetzky S, Werfel T, Rösner LM. Autoallergy in atopic dermatitis. Allergo J Int. 2015;24:16-22.

68. Megna M, Napolitano M, Patruno C, Villani A, Balato A, Monfrecola G, et al. Systemic treatment of adult atopic dermatitis: a review. Dermatol Ther (Heidelb). 2017;7:1-23.

69. Yu SH, Drucker AM, Lebwohl M, Silverberg JI. A systematic review of the safety and efficacy of systemic corticosteroids in atopic dermatitis. J Am Acad Dermatol. 2018;78:733-40.

70. Drucker AM, Eyerich K, de Bruin-Weller MS, Thyssen JP, Spuls PI, Irvine AD, et al. Use of systemic corticosteroids for atopic dermatitis: International Eczema Council consensus statement. Br J Dermatol. 2018;178:768-75.

71. Jackson SM, Nesbitt LT. Glucocorticosteroids. In: Bolognia JL, Jorizzo JL, Schaffer JV, editors. Dermatology, vol. 1. 3rd ed. Amsterdam: Elsevier; 2012. p. 2075-88.

72. Furue M. Topical corticosteroids: how to use in clinical practice (in Japanese). Arerugi. 2009;58:491-8.

73. Aso M. The effects of potent topical corticosteroids on adrenocortical function. J Dermatol. 1983;10:145-9.

74. Vestergaard P, Rejnmark L, Mosekilde L. Fracture risk associated with systemic and topical corticosteroids. J Intern Med. 2005;257:374-84.

75. Suzuki Y, Nawata H, Soen S, Fujiwara S, Nakayama H, Tanaka I, et al. Guidelines on the management and treatment of glucocorticoid-induced osteoporosis of the Japanese Society for Bone and Mineral Research: 2014 update. J Bone Miner Metab. 2014;32:337-50.

76. Reddy KR, Beavers KL, Hammond SP, Lim JK, Falck-Ytter YT. American Gastroenterological Association Institute guideline on the prevention and treatment of hepatitis B virus reactivation during immunosuppressive drug therapy. Gastroenterology. 2015;148:215-9.

77. Aguirre R, May JM. Inflammation in the vascular bed: importance of vitamin C. Pharmacol Ther. 2008;119:96-103.

78. Ogawa S, Oka M, Kunisada M, Nishigori C. Unilateral lower leg purpura. Dermatol Online J. 2013;19(1):16.

79. Fisher DA. Adverse effects of topical corticosteroid use. West J Med. 1995;162:123-6.

80. Dezoteux F, Astrid B, Chuffard M, Drumez E, Azib S, StaumontSallé D. Atopic dermatitis in elderly adults. Eur J Dermatol. 2019;29(4):371-4. https://doi.org/10.1684/ejd.2019.3609.

81. Garritsen FM, van der Schaft J, van den Reek JM, Politiek K, van Os-Medendorp H, van Dijk M, et al. Risk of non-melanoma skin cancer in patients with atopic dermatitis treated with oral immunosuppressive drugs. Acta Derm Venereol. 2017;97:724-30.

82. Sinha A, Velangi S, Natarajan S. Non-Hodgkin's lymphoma following treatment of atopic eczema with cyclosporin A. Acta Derm Venereol. 2004;84:327-8.

83. van der Schaft J, van Zuilen AD, Deinum J, Bruijnzeel-Koomen CA, de Bruin-Weller MS. Serum creatinine levels during and after long-term treatment with cyclosporine A in patients with severe atopic dermatitis. Acta Derm Venereol. 2015;95:963-7.
84. Fulop T, Larbi A, Kotb R, de Angelis F, Pawelec G. Aging, immunity, and cancer. Discov Med. 2011;11(61):537-50.

85. Tanei R, Oda A, Hasegawa Y. Narrow-band ultraviolet B is a useful adjunctive treatment for atopic dermatitis in older adults: case reports. Dermatol Clin Res. 2016;2(3):112-7.

86. Tintle S, Shemer A, Suárez-Fariñas M, Fujita H, Gilleaudeau P, Sullivan-Whalen M, et al. Reversal of atopic dermatitis with narrow-band UVB phototherapy and biomarkers for therapeutic response. J Allergy Clin Immunol. 2011;128:583-93.

87. Beck LA, Thaçi D, Hamilton JD, Graham NM, Bieber T, Rocklin $\mathrm{R}$, et al. Dupilumab treatment in adults with moderate-to-severe atopic dermatitis. N Engl J Med. 2014;371:130-9.

88. Hamilton JD, Ungar B, Guttman-Yassky E. Drug evaluation review: dupilumab in atopic dermatitis. Immunotherapy. 2015;7:1043-58

89. Hamilton JD, Suárez-Fariñas M, Dhingra N, Cardinale I, Li X, Kostic A, Ming JE, et al. Dupilumab improves the molecular signature in skin of patients with moderate-to-severe atopic dermatitis. J Allergy Clin Immunol. 2014;134:1293-300.

90. Guttman-Yassky E, Bissonnette R, Ungar B, Suárez-Fariñas M, Ardeleanu M, Esaki H, et al. Dupilumab progressively improves systemic and cutaneous abnormalities in patients with atopic dermatitis. J Allergy Clin Immunol. 2019;143:155-72.

91. de Bruin-Weller M, Thaçi D, Smith CH, Reich K, Cork MJ, Radin A, et al. Dupilumab with concomitant topical corticosteroid treatment in adults with atopic dermatitis with an inadequate response or intolerance to ciclosporin $\mathrm{A}$ or when this treatment is medically inadvisable: a placebo-controlled, randomized phase III clinical trial (LIBERTY AD CAFÉ). Br J Dermatol. 2018;178:1083-101.

92. Castro M, Corren J, Pavord ID, Maspero J, Wenzel S, Rabe $\mathrm{KF}$, et al. Dupilumab efficacy and safety in moderate-to-severe uncontrolled asthma. N Engl J Med. 2018;378:2486-96.

93. Wollenberg A, Ariens L, Thurau S, van Luijk C, Seegräber M, de Bruin-Weller M. Conjunctivitis occurring in atopic dermatitis patients treated with dupilumab-clinical characteristics and treatment. J Allergy Clin Immunol Pract. 2018;6:1778-80.

94. Fukuda K, Ishida W, Kishimoto T, Fukushima A. Development of conjunctivitis with a conjunctival proliferative lesion in a patient treated with dupilumab for atopic dermatitis. Allergol Int. 2019;68:383-4.

95. Waldman RA, DeWane ME, Sloan B, Grant-Kels JM. Characterizing dupilumab facial redness: a multi-institution retrospective medical record review. J Am Acad Dermatol. 2020;82(1):230-2.

96. de Beer FSA, Bakker DS, Haeck I, Ariens L, van der Schaft J, van Dijk MR, et al. Dupilumab facial redness: positive effect of itraconazole. JAAD Case Rep. 2019;5:888-91.

97. Suresh R, Murase JE. The role of expanded series patch testing in identifying causality of residual facial dermatitis following initiation of dupilumab therapy. JAAD Case Rep. 2018;4:899-904.

98. Paller AS, Tom WL, Lebwohl MG, Blumenthal RL, Boguniewicz M, Call RS, et al. Efficacy and safety of crisaborole ointment, a novel, nonsteroidal phosphodiesterase 4 (PDE4) inhibitor for the topical treatment of atopic dermatitis (AD) in children and adults. J Am Acad Dermatol. 2016;75:494-503.

99. Fabbrocini G, Napolitano M, Megna M, Balato N, Patruno C. Treatment of atopic dermatitis with biologic drugs. Dermatol Ther (Heidelb). 2018;8:527-38.

100. Czarnowicki T, He H, Krueger JG, Guttman-Yassky E. Atopic dermatitis endotypes and implications for targeted therapeutics. J Allergy Clin Immunol. 2019;143:1-11. 